\title{
nature
protocols
}

\section{Author Correction: A practical guide to intelligent image-activated cell sorting}

Akihiro Isozaki, Hideharu Mikami, Kotaro Hiramatsu, Shinya Sakuma (D), Yusuke Kasai D, Takanori lino,

Takashi Yamano, Atsushi Yasumoto, Yusuke Oguchi, Nobutake Suzuki, Yoshitaka Shirasaki, Taichiro Endo,

Takuro Ito, Kei Hiraki, Makoto Yamada, Satoshi Matsusaka, Takeshi Hayakawa, Hideya Fukuzawa, Yutaka Yatomi,

Fumihito Arai, Dino Di Carlo, Atsuhiro Nakagawa, Yu Hoshino (iD, Yoichiroh Hosokawa, Sotaro Uemura,

Takeaki Sugimura, Yasuyuki Ozeki (D), Nao Nitta (D) and Keisuke Goda

Correction to: Nature Protocols https://doi.org/10.1038/s41596-019-0183-1, published online 5 July 2019.

The format of the files for Supplementary Data 1 and Supplementary Data 2 initially published online was incorrect. The files were replaced on 17 October 2019.

Published online: 17 October 2019

https://doi.org/10.1038/s41596-019-0252-5 